\title{
IS-2: Simulating Bacterial Growth, Competition, and Resistance with Agent-Based Models and Laboratory Experiments
}

\author{
Anne E. Yust*, Davida S. Smyth \\ Department of Natural Sciences \& Mathematics, Eugene Lang College of Liberal Arts at The New School, New \\ York, NY 10011 \\ yusta@newschool.edu
}

Antibiotic resistance is a capacious and global problem, considered to be one of the most important public health threats of the 21st century. In our proposed chapter, we review the basic biological and mathematical concepts underlying the phenomenon. We then provide tutorial-type instruction to build agent-based modeling skills, ultimately to simulate and analyze antibiotic resistance and its effect on the planet. In this talk, we will summarize the content of our proposed chapter on undergraduate research that outlines how students can use agent-based models together with laboratory experiments to explore bacteria growth, competition, and resistance. Then, we'll provide explicit examples of problems and projects posed throughout the chapter. We will discuss how both simulation and laboratory-based exercises can be used to deepen student understanding and provide richer research experience. 